\title{
CARACTERIZAÇÃO DE AGREGADOS EM UM LATOSSOLO VERMELHO DISTROFÉRRICO TÍPICO SUBMETIDO A DIFERENTES DOSES DE VINHAÇA ${ }^{(1)}$
}

\author{
Amarildo Luiz Passarin ${ }^{(2)}$, Edinei Luis Rodrigueiro ${ }^{(3)}$, Carla Renata \\ Pazzotti Robaina ${ }^{(4)} \&$ Cristiane de Conti Medina ${ }^{(5)}$
}

\begin{abstract}
RESUMO
A distribuição de classes de tamanho, a quantidade e a estabilidade dos agregados constituem importantes critérios para avaliação da estrutura, a qual desempenha papel relevante nas relações entre o solo e as plantas. A vinhaça, quando aplicada ao solo, provoca aumento na atividade microbiana em conseqüência da adição da matéria orgânica nela contida. Essa atividade é acompanhada pelo aumento na produção de mucilagem, que pode favorecer a agregação e promover aumento na estabilidade estrutural do solo. $O$ objetivo deste trabalho foi avaliar a estabilidade de agregados em um Latossolo Vermelho distroférrico típico textura muito argilosa tratado com diferentes doses de vinhaça. O experimento foi conduzido na Fazenda-Escola da Universidade Estadual de Londrina no período de agosto de 1997 a agosto de 1999, num delineamento de blocos ao acaso com cinco tratamentos (doses de vinhaça) e quatro repetições. Amostras de solo foram coletadas nas profundidades de 0-10, 10-20, 20-30 e 30$40 \mathrm{~cm}$. As avaliações do presente trabalho são referentes à quinta colheita (quarta soca), realizada em agosto de 1999. As doses de vinhaça foram de $0,150,300,450 \mathrm{e}$ $600 \mathrm{~m}^{3} \mathrm{ha}^{-1}$, e os índices de agregação determinados foram diâmetro médio ponderado (DMP), diâmetro médio geométrico (DMG) e índice de estabilidade de agregados (IEA). Os resultados mostraram que as diferentes doses de vinhaça não promoveram mudanças significativas nos índices de agregação nas profundidades estudadas.
\end{abstract}

Termos de indexação: física do solo, índices de agregação, matéria orgânica.

\footnotetext{
(1) Parte da Tese de Doutorado do terceiro autor apresentada à Faculdade de Ciências Agronômicas, Universidade Estadual Paulista - UNESP. Recebido para publicação em fevereiro de 2005 e aprovado em julho de 2007.

${ }^{(2)}$ Mestrando do Programa de Pós-Graduação em Agronomia, Universidade Estadual de Londrina - UEL. Caixa Postal 6001, CEP86051-990 Londrina (PR). E-mail: amarildoluizp@yahoo.com.br

${ }^{(3)}$ Engenheiro-Agrônomo. Agroamazônia. Av. Tenente Coronel Duarte 1777, CEP 78015-501 Cuiabá (MG).

(4) Engenheira-Agrônoma. Rua Venezuela 41, CEP 86010-680 Londrina (PR). E-mail: crrobaiana@hotmail.com

${ }^{(5)}$ Professora do Departamento de Agronomia, UEL. E-mail: medina@uel.br
} 


\title{
SUMMARY: EVALUATION OF AGGREGATE STABILITY IN AN OXISOL TREATED WITH DIFFERENT VINASSE DOSES
}

\begin{abstract}
The size distribution, the quantity and stability of soil aggregates are important parameters to evaluate the soil structure, which plays a relevant role in soil-plant relationships. Vinasse when applied on soil stimulates microbial activity because of its organic matter content. This activity may increase mucilage production and improve the aggregation and soil structural stability. The objective of this study was to evaluate the aggregate stability in an Oxisol treated with different vinasse doses. The study was carried out at the Londrina State University Exerimental Farm from August 1997 to August 1999, in randomized blocks, with five treatments (vinasse doses) and four replications. The soil samples were collected in four soil layers $(0-10,10-20,20-30$ and $30-40 \mathrm{~cm})$. The evaluations of this study were carried out at the fifth harvest (fourth ratoon), in August 1999. Vinasse was applied at 0, 150, 300, 450 and $600 \mathrm{~m}^{3} \mathrm{ha}^{-1}$ and the following aggregation indices were determined: Ponderate Middle Diameter (PMD), Geometrical Middle Diameter (GMD) and Aggregate Stability Index (ASI). The results showed that different vinasse doses did not significantly alter the aggregation indices of the different depths studied.
\end{abstract}

Index terms: soil physics, aggregation indices, organic matter.

\section{INTRODUÇÃO}

De acordo com Kiehl (1979), para haver formação dos agregados no solo são necessárias duas condições fundamentais: primeira, que uma força mecânica qualquer provoque a aproximação das partículas do solo - esse movimento pode ser causado pelo crescimento das raízes, por animais de hábito terrestre, pelo fenômeno da expansão e contração do solo provocado pelo molhamento e secamento alternado, ou pela floculação; a segunda condição é a de que, após o contato das partículas, haja um agente cimentante para consolidar essa união, gerando o agregado.

A matéria orgânica, os minerais de argila e os óxidos de $\mathrm{Fe}$ são os agentes cimentantes que mais contribuem para a agregação do solo. A matéria orgânica é mais eficiente na formação de agregados estáveis, e isso se deve à contribuição da atividade microbiana, à fauna terrestre e à vegetação, fatores que se associam à presença da matéria orgânica no solo (Kiehl, 1979). Com o incremento do teor de matéria orgânica há melhor distribuição de micro e macroporos do solo, favorecendo sua aeração e capacidade de infiltração e retenção de água. Além disso, há melhor estruturação e redução da densidade do solo, favorecendo a exploração de maior volume de solo pelas raízes (Longo, 1994).

Tisdall \& Oades (1982) mostraram que a matéria orgânica exerce papel importante na formação e estabilização dos agregados do solo devido a ligações entre polímeros orgânicos e a superfície inorgânica, mediadas por cátions polivalentes. Baver \& Gardner (1972) observou correlação direta entre a percentagem de agregados maiores que $0,1 \mathrm{~mm}$, estáveis em água, e o teor de carbono orgânico de vários solos. Além disso, o autor encontrou correlações entre o teor de carbono orgânico e a agregação em solos com menos de $25 \%$ de argila, corroborando, assim, com os dados obtidos por Carpenedo \& Mielniczuck (1990). Ademais, Castro Filho \& Logan (1991) afirmam que os mecanismos de formação das diferentes classes de tamanho dos agregados são influenciados pela matéria orgânica, cuja quantidade irá permitir maior ou menor agregação, resultando em menor ou maior perda de solo.

Ao avaliar a agregação dos solos, o interesse agronômico volta-se para a distribuição de tamanhos, quantidade e estabilidade dos agregados, pois esses fatores de agregação são importantes na determinação da quantidade e distribuição dos espaços porosos e, ainda, na suscetibilidade dos agregados à ação erosiva da água e do vento (Baver et al., 1973).

O tamanho dos agregados e o estado de agregação do solo podem ser determinados de várias formas. Segundo Kemper (1965) e Kemper \& Chepil (1965), podem ser usados como parâmetros o diâmetro médio ponderado (DMP), o diâmetro médio geométrico (DMG) e o índice de estabilidade dos agregados (IEA), que são obtidos pelo fracionamento do solo em classes de agregados, por meio de tamisamento.

Cada parâmetro apresenta um princípio diferente: o DMP é tanto maior quanto maior for a percentagem de agregados grandes retidos nas peneiras com malhas maiores; o DMG representa uma estimativa do tamanho da classe de agregados de maior ocorrência; e o IEA representa uma medida da agregação total do solo e não considera a distribuição por classe de agregados (Castro Filho et al., 1998).

A agregação das partículas de solo tratado com vinhaça por longo tempo se dá, principalmente, em função da mucilagem excretada pelos microrganismos que metabolizam açúcares de cadeia pequena, presentes em grande quantidade na vinhaça, de tal 
forma que altas taxas de aplicação do resíduo juntamente com o tempo adequado de contato com o solo favoreceriam a aglutinação das partículas, promovendo aumento na sua estabilidade estrutural (Camargo et al., 1983). De fato, estes autores constataram que a agregação do solo em uma área que recebeu vinhaça ao longo do tempo foi maior que na área onde não houve sua aplicação. Aguiar (1992), por sua vez, encontrou resultados positivos com relação à estabilidade estrutural em dois solos, um arenoargiloso e outro argilo-siltoso, tratados com vinhaça, e Longo (1994), avaliando os efeitos da vinhaça sobre os atributos físicos, químicos e bioquímicos de um Latossolo Vermelho-Amarelo textura média, também constatou que o tratamento com vinhaça promoveu aumento na estabilidade de agregados do solo.

Sabe-se que a vinhaça é um subproduto gerado a partir da produção do álcool. Sua produção é da ordem de 12 a $13 \mathrm{~L}$ por litro de álcool produzido, o que representa grande volume de resíduo (Medina \& Brinholi, 1998). Contudo, sua elevada demanda bioquímica de oxigênio inviabiliza seu lançamento nos cursos d'água; assim, justificam-se os esforços visando seu aproveitamento para os mais diversos fins (Aguiar et al., 1992).

O objetivo deste trabalho foi avaliar a estabilidade de agregados de um Latossolo Vermelho distroférrico típico textura muito argilosa tratado com diferentes doses de vinhaça in natura.

\section{MATERIAL E MÉTODOS}

Em outubro de 1994, na Fazenda-Escola da Universidade Estadual de Londrina, localizada no município de Londrina-PR, $23^{\circ} 23^{\prime}$ ' S e $51^{\circ} 11^{\prime} \mathrm{W}$ e $566 \mathrm{~m}$ de altitude, foi feito o plantio da variedade de cana-de-açúcar RB 785148, cujas características são: maturação média/tardia, boa capacidade de perfilhamento, alta produtividade agrícola, médio teor de sacarose, bom comportamento em solos de baixa fertilidade, alta resistência à ferrugem e reação intermediária ao carvão, mosaico e escaldadura das folhas (Programa..., 1988).

O clima característico da região, de acordo com a classificação de Köppen, é Subtropical Úmido Mesotérmico, apresentando temperatura média no mês mais frio inferior a $18^{\circ} \mathrm{C}$ e temperatura média no mês mais quente acima de $22^{\circ} \mathrm{C}$, verões quentes, geadas pouco freqüentes e tendência de concentração das chuvas nos meses de verão, sem estação seca definida. A precipitação média anual varia de 1.400 a $1.600 \mathrm{~mm}$; a temperatura média anual varia de 21 a $22{ }^{\circ} \mathrm{C}$; e a umidade relativa anual, de 75 a $80 \%$ (IAPAR, 2004).

O solo, classificado no atual Sistema Brasileiro de Classificação (Embrapa, 1999) como Latossolo Vermelho distroférrico típico textura muito argilosa, foi preparado com arado de discos a uma profundidade de $30 \mathrm{~cm}$, seguido de duas gradagens e da passagem de sulcador para abertura dos sulcos. Como adubação básica foram utilizados: $20 \mathrm{~kg} \mathrm{ha}^{-1} \mathrm{de} \mathrm{N}, 90 \mathrm{~kg} \mathrm{ha}^{-1}$ de $\mathrm{P}_{2} \mathrm{O}_{5}$ e $90 \mathrm{~kg} \mathrm{ha}^{-1}$ de $\mathrm{K}_{2} \mathrm{O}$, empregando-se, respectivamente, uréia, superfosfato simples e cloreto de potássio; 60 dias após o plantio, foram aplicados $50 \mathrm{~kg} \mathrm{ha}^{-1}$ de uréia como fonte de $\mathrm{N}$.

O delineamento experimental utilizado foi o de blocos ao acaso, com cinco tratamentos e quatro repetições, sendo cada parcela composta de cinco linhas com $8 \mathrm{~m}$ de comprimento, espaçadas de 1,20 m entre si. Para as avaliações foram consideradas apenas as três linhas centrais de cada parcela, suprimindose $1,50 \mathrm{~m}$ em cada extremidade.

As doses de vinhaça foram: $0 ; 150 ; 300 ; 450$; e $600 \mathrm{~m}^{3} \mathrm{ha}^{-1}$. As doses foram aplicadas em dezembro de 1997 e de 1998, após a terceira e a quarta colheitas (segunda e terceira socas), e as avaliações foram realizadas em agosto de 1999, após a quinta colheita (quarta soca). Tanto as aplicações da vinhaça quanto as colheitas e as capinas foram realizadas manualmente.

Para determinar a composição química da vinhaça de caldo misto, a extração do $\mathrm{N}$ foi feita através da digestão sulfúrica, e sua determinação, pelo método de Kjeldahl; as extrações de P, K, Ca, Mg e S foram feitas por meio da digestão nítrico-perclórica; o teor de $\mathrm{P}$ foi determinado por espectrofotometria com molibdato de amônio; o K, por fotometria de chama; o Ca e o Mg, por espectrofotometria de absorção atômica; e o S, por espectrofotometria com precipitação de cloreto de bário, conforme método descrito por Kiehl (1985). A composição química média, em $\mathrm{kg} \mathrm{m}^{-3}$, de $\mathrm{N}, \mathrm{P}, \mathrm{K}$, $\mathrm{Ca}, \mathrm{Mg}$ e S foi, respectivamente, de 0,28; 0,052; 1,992; 1,$10 ; 0,24 ;$ e 5,48 .

As amostras de solo foram coletadas em trincheiras de $1,50 \times 1,00 \mathrm{~m}$, transversais às linhas de plantio, nas seguintes profundidades: $0-10,10-20,20-30$ e $30-40 \mathrm{~cm}$. Após coleta, o solo foi seco à sombra, para retirada do excesso de umidade. Para homogeneização da amostra, o volume total de cada uma delas foi passado em peneira com malha de $19 \mathrm{~mm}$, e os torrões com diâmetros maiores que as malhas da peneira foram quebrados nos seus pontos de fraqueza. Depois de peneirada, a amostra de solo foi imediatamente acondicionada em sacos plásticos, para evitar perda posterior de umidade.

No laboratório, cada amostra de solo foi subdividida em quatro subamostras, cada qual com $40 \mathrm{~g}$. Uma parte foi submetida à secagem em estufa a $105^{\circ} \mathrm{C}$, para determinação da umidade e massa seca da amostra. As demais subamostras foram utilizadas no processo de umedecimento e tamisagem, de acordo com Yoder (1936).

A distribuição das classes de agregados para cada amostra de solo foi determinada em um tamisador. Cada conjunto no tamisador foi constituído de seis peneiras, cujas malhas tinham as seguintes aberturas: 8, 4, 2, 1, 0,5 e 0,25 mm. Quantificou-se o 
solo seco retido em cada peneira e, ainda, aquele que passou pela última peneira $(<0,25 \mathrm{~mm})$, obtendo-se sete diferentes classes de agregados.

Sobre a peneira de maior abertura de malha de cada conjunto de peneiras, que continha papel-filtro para retenção da terra, foram colocados $40 \mathrm{~g}$ da amostra de solo. O umedecimento da amostra deu-se por capilaridade através da elevação do nível de água no interior do tamisador até que ela tocasse o papelfiltro, promovendo o molhamento do solo por um período de 15 min (Santos, 1993). Depois da saturação da amostra, o papel-filtro foi retirado e o tamisador acionado por $10 \mathrm{~min}$.

Posteriormente, as peneiras foram retiradas do tamisador, e os agregados retidos em cada uma delas foram transferidos para frascos que, em seguida, foram colocados em estufa a $105^{\circ} \mathrm{C}$ por $24 \mathrm{~h}$, para secagem. Depois de secas, as amostras foram pesadas novamente. Com a obtenção da massa de solo seco de cada classe de agregados, calculou-se, então, sua média. A classe de agregados de solo mais fino foi considerada aquela em que o solo passou pela peneira de malha de $0,25 \mathrm{~mm}$, ficando disperso no tamisador. Seu valor foi calculado pela diferença entre o somatório do peso das outras classes de agregados e o peso total da amostra seca. Com os valores de todos os dados obtidos, foram calculados o DMP, o DMG e o IEA, de acordo com a planilha de cálculos proposta por Castro Filho et al. (1998).

Os resultados foram submetidos à análise de variância e as médias dos tratamentos comparadas pelo teste de Tukey a $5 \%$.

\section{RESULTADOS E DISCUSSÃO}

Pelos resultados da análise de variância, verificouse que as diferentes doses de vinhaça não promoveram aumentos significativos nos índices de agregação para as profundidades estudadas. Do mesmo modo, Resende (1979), estudando os efeitos de doses crescentes de vinhaça sobre algumas propriedades de um solo aluvial, constatou que não houve alterações no estado de agregação do solo, e Aguiar et al. (1992), ao estudarem um Latossolo Roxo e um Latossolo Vermelho-Escuro distrófico, também observaram que houve pequeno ou nenhum efeito das doses de vinhaça sobre as características físicas dos solos.

Esses resultados podem ser atribuídos à quantidade de material orgânico contido na vinhaça natural, que não é suficiente para promover a agregação das partículas do solo, ou, por ser altamente biodegradável, não consegue atuar como agente cimentante (Longo et al., 1996). Além disso, a matéria orgânica em si, sem transformações biológicas, tem efeito muito pequeno, se é que tem algum, na estrutura do solo (Baver et al., 1973). Sua influência na agregação do solo é um processo dinâmico, e seus efeitos benéficos são o resultado da atividade conjunta dos microrganismos, da fauna e da vegetação (Campos et al., 1995). Desse modo, o tempo de contato da vinhaça com o solo e a quantidade e as espécies de microrganismos podem não ter sido suficientes para ocorrência das interações necessárias entre os fatores bióticos e a realização de uma atividade biológica capaz de promover melhorias na estabilidade dos agregados.

Nos quadros 1 e 2 pode-se verificar que não houve redução na quantidade de agregados nas classes de maior tamanho, nem concentração nas classes de menor diâmetro, para as diferentes doses e profundidades. Esses resultados sugerem que não ocorreu fracionamento dos agregados em unidades menores. De fato, na área do experimento não houve revolvimento de solo nem tráfego de máquinas ou implementos agrícolas, fatores que promovem modificações no tamanho dos agregados do solo (Cintra, 1980; Silva, 1980).

Embora não tenha havido diferenças significativas, é possível observar que, em relação ao DMP, os maiores valores obtidos foram para as doses de $150 \mathrm{~m}^{3} \mathrm{ha}^{-1} \mathrm{na}$ profundidade de $30-40 \mathrm{~cm}$ e de $300 \mathrm{~m}^{3} \mathrm{ha}^{-1} \mathrm{na}$ profundidade de $20-30 \mathrm{~cm}$. Nas profundidades de $0-10$ e $10-20 \mathrm{~cm}$, todos os valores foram inferiores aos da testemunha. Para a profundidade de $30-40 \mathrm{~cm}$, todos os valores foram superiores em relação a ela.

No que se refere ao DMG, o maior valor foi observado para a dose de $300 \mathrm{~m}^{3} \mathrm{ha}^{-1}$, na profundidade de $0-10 \mathrm{~cm}$. Na profundidade de $10-20 \mathrm{~cm}$, todos os valores observados ficaram abaixo dos da testemunha. Quanto à profundidade de $20-30 \mathrm{~cm}$, a dose que proporcionou maior DMG foi de $150 \mathrm{~m}^{3} \mathrm{ha}^{-1}$, e para $30-40 \mathrm{~cm}$, a de $600 \mathrm{~m}^{3} \mathrm{ha}^{-1}$.

Em relação ao IEA, os maiores valores foram obtidos para a dose de $150 \mathrm{~m}^{3} \mathrm{ha}^{-1}$, nas profundidades de 0-10 e 20-30 cm. Contudo, independentemente das doses e das profundidades, os valores são semelhantes e retratam o bom índice de agregação total do solo. Esses dados ratificam a afirmação de Carpenedo \& Mielniczuk (1990), segundo a qual, em seu estado natural, os Latossolos caracterizam-se pela boa estabilidade estrutural, pois, além da matéria orgânica, apresentam elevados teores de argila e óxidos de $\mathrm{Fe}$ e Al, que, de acordo com Baver et al. (1973) e Kiehl (1979), atuam como agentes de estabilização dos agregados. Ademais, o experimento foi realizado em uma área cultivada com cana-de-açúcar, que é uma gramínea semiperene. Nesse sentido, muitos pesquisadores têm destacado os benefícios dessa família de plantas na formação e estabilização dos agregados do solo. Esses efeitos benéficos são atribuídos, principalmente, à alta densidade de raízes, que promove a aproximação de partículas pela constante absorção de água do solo, às periódicas renovações do sistema radicular e à uniforme distribuição dos exsudatos no solo, que estimulam a atividade microbiana, cujos subprodutos atuam na formação e estabilização dos agregados (Silva \& Mielniczuk, 1997). 
Quadro 1. Diâmetro médio ponderado (DMP), diâmetro médio geométrico (DMG) e índice de estabilidade de agregados (IEA) em quatro profundidades de um Latossolo Vermelho tratado com cinco doses de vinhaça

\begin{tabular}{|c|c|c|c|c|}
\hline Profundidade & Dose & DMP & DMG & IEA \\
\hline $\mathrm{cm}$ & $\mathrm{m}^{3} \mathrm{ha}^{-1}$ & \multicolumn{2}{|c|}{$\mathrm{mm}$} & $\%$ \\
\hline $0-10$ & $\begin{array}{r}0 \\
150 \\
300 \\
450 \\
600\end{array}$ & $\begin{array}{l}3,43 \mathrm{a} \\
3,30 \mathrm{a} \\
3,41 \mathrm{a} \\
2,73 \mathrm{a} \\
3,08 \mathrm{a}\end{array}$ & $\begin{array}{l}1,31 \mathrm{a} \\
1,23 \mathrm{a} \\
2,02 \mathrm{a} \\
1,01 \mathrm{a} \\
1,05 \mathrm{a}\end{array}$ & $\begin{array}{l}81,78 \mathrm{a} \\
83,98 \mathrm{a} \\
80,86 \mathrm{a} \\
82,77 \mathrm{a} \\
80,67 \mathrm{a}\end{array}$ \\
\hline $10-20$ & $\begin{array}{r}0 \\
150 \\
300 \\
450 \\
600\end{array}$ & $\begin{array}{l}3,63 \mathrm{a} \\
3,28 \mathrm{a} \\
3,15 \mathrm{a} \\
2,64 \mathrm{a} \\
2,91 \mathrm{a}\end{array}$ & $\begin{array}{l}1,39 \mathrm{a} \\
1,26 \mathrm{a} \\
1,05 \mathrm{a} \\
1,17 \mathrm{a} \\
1,12 \mathrm{a}\end{array}$ & $\begin{array}{l}75,81 \mathrm{a} \\
79,64 \mathrm{a} \\
73,76 \mathrm{a} \\
80,82 \mathrm{a} \\
79,44 \mathrm{a}\end{array}$ \\
\hline $20-30$ & $\begin{array}{r}0 \\
150 \\
300 \\
450 \\
600\end{array}$ & $\begin{array}{l}3,40 \mathrm{a} \\
3,56 \mathrm{a} \\
3,83 \mathrm{a} \\
2,67 \mathrm{a} \\
3,13 \mathrm{a}\end{array}$ & $\begin{array}{l}1,41 \mathrm{a} \\
1,74 \mathrm{a} \\
1,48 \mathrm{a} \\
0,86 \mathrm{a} \\
1,22 \mathrm{a}\end{array}$ & $\begin{array}{l}77,30 \mathrm{a} \\
87,04 \mathrm{a} \\
72,16 \mathrm{a} \\
65,97 \mathrm{a} \\
77,43 \mathrm{a}\end{array}$ \\
\hline $30-40$ & $\begin{array}{r}0 \\
150 \\
300 \\
450 \\
600\end{array}$ & $\begin{array}{l}3,20 \mathrm{a} \\
3,94 \mathrm{a} \\
3,31 \mathrm{a} \\
3,42 \mathrm{a} \\
3,52 \mathrm{a}\end{array}$ & $\begin{array}{l}1,42 \mathrm{a} \\
1,56 \mathrm{a} \\
1,37 \mathrm{a} \\
1,54 \mathrm{a} \\
1,65 \mathrm{a}\end{array}$ & $\begin{array}{l}73,51 \mathrm{a} \\
68,35 \mathrm{a} \\
69,89 \mathrm{a} \\
80,77 \mathrm{a} \\
80,35 \mathrm{a}\end{array}$ \\
\hline CV (\%) & & 12,03 & 16,52 & 7,14 \\
\hline
\end{tabular}

(1) Valores médios seguidos de letras minúsculas iguais na coluna, para cada profundidade do solo e cada profundidade, não diferem estatisticamente entre si pelo teste de Tukey a $5 \%$.

Quadro 2. Massa dos agregados secos distribuídos em sete classes de tamanho em função de quatro profundidades de um Latossolo Vermelho tratado com cinco doses de vinhaça

\begin{tabular}{|c|c|c|c|c|c|c|c|c|}
\hline \multirow{2}{*}{ Profundidade } & \multirow{2}{*}{ Dose } & \multicolumn{7}{|c|}{ Classe de tamanho do agregado (mm) } \\
\hline & & $>8$ & $8-4$ & $4-2$ & $2-1$ & $1-0,5$ & $0,5-0,25$ & $<0,25$ \\
\hline $\mathrm{cm}$ & $\mathrm{m}^{3} \mathrm{ha}^{-1}$ & \multicolumn{7}{|c|}{ Massa dos agregados, $\mathrm{g} / 40 \mathrm{~g}^{(1)}$} \\
\hline $0-10$ & $\begin{array}{r}0 \\
150 \\
300 \\
450 \\
600\end{array}$ & $\begin{array}{l}5,08 \mathrm{a} \\
4,90 \mathrm{a} \\
4,63 \mathrm{a} \\
3,70 \mathrm{a} \\
4,75 \mathrm{a}\end{array}$ & $\begin{array}{l}6,80 \text { a } \\
6,46 \text { a } \\
5,25 \text { a } \\
5,10 \text { a } \\
5,43 \text { a }\end{array}$ & $\begin{array}{l}4,20 \mathrm{a} \\
3,76 \mathrm{a} \\
5,10 \mathrm{a} \\
3,95 \mathrm{a} \\
4,00 \mathrm{a}\end{array}$ & $\begin{array}{l}4,63 \mathrm{a} \\
4,46 \mathrm{a} \\
3,13 \mathrm{a} \\
4,83 \mathrm{a} \\
4,38 \mathrm{a}\end{array}$ & $\begin{array}{l}4,95 \mathrm{a} \\
5,31 \mathrm{a} \\
4,30 \mathrm{a} \\
5,25 \mathrm{a} \\
4,57 \mathrm{a}\end{array}$ & $\begin{array}{r}7,20 \mathrm{a} \\
8,80 \mathrm{a} \\
10,13 \mathrm{a} \\
10,28 \mathrm{a} \\
9,33 \mathrm{a}\end{array}$ & $\begin{array}{l}7,14 \text { a } \\
6,31 \mathrm{a} \\
7,46 \mathrm{a} \\
6,89 \mathrm{a} \\
7,54 \mathrm{a}\end{array}$ \\
\hline $10-20$ & $\begin{array}{r}0 \\
150 \\
300 \\
450 \\
600\end{array}$ & $\begin{array}{l}5,50 \mathrm{a} \\
4,80 \mathrm{a} \\
5,15 \mathrm{a} \\
4,00 \mathrm{a} \\
4,12 \mathrm{a}\end{array}$ & $\begin{array}{l}7,13 \text { a } \\
6,37 \text { a } \\
5,65 \text { a } \\
4,12 \text { a } \\
5,75 \text { a }\end{array}$ & $\begin{array}{l}4,53 \mathrm{a} \\
3,96 \mathrm{a} \\
3,23 \mathrm{a} \\
3,58 \mathrm{a} \\
3,68 \mathrm{a}\end{array}$ & $\begin{array}{l}4,73 \mathrm{a} \\
4,68 \mathrm{a} \\
3,61 \mathrm{a} \\
4,38 \mathrm{a} \\
4,19 \mathrm{a}\end{array}$ & $\begin{array}{l}4,72 \mathrm{a} \\
6,53 \mathrm{a} \\
5,21 \mathrm{a} \\
5,30 \mathrm{a} \\
4,12 \mathrm{a}\end{array}$ & $\begin{array}{r}4,10 \mathrm{a} \\
6,20 \mathrm{a} \\
7,18 \mathrm{a} \\
11,31 \mathrm{a} \\
10,45 \mathrm{a}\end{array}$ & $\begin{array}{l}9,29 \mathrm{a} \\
7,46 \mathrm{a} \\
9,97 \mathrm{a} \\
7,31 \mathrm{a} \\
7,69 \mathrm{a}\end{array}$ \\
\hline $20-30$ & $\begin{array}{r}0 \\
150 \\
300 \\
450 \\
600\end{array}$ & $\begin{array}{l}5,00 \mathrm{a} \\
5,11 \mathrm{a} \\
6,31 \mathrm{a} \\
4,43 \mathrm{a} \\
4,78 \mathrm{a}\end{array}$ & $\begin{array}{l}6,81 \text { a } \\
6,95 \text { a } \\
6,81 \text { a } \\
3,58 \text { a } \\
5,68 \text { a }\end{array}$ & $\begin{array}{l}4,28 \mathrm{a} \\
4,56 \mathrm{a} \\
4,45 \mathrm{a} \\
4,03 \mathrm{a} \\
3,80 \mathrm{a}\end{array}$ & $\begin{array}{l}4,71 \mathrm{a} \\
5,58 \mathrm{a} \\
4,96 \mathrm{a} \\
3,41 \mathrm{a} \\
4,55 \mathrm{a}\end{array}$ & $\begin{array}{l}4,38 \mathrm{a} \\
8,42 \mathrm{a} \\
4,65 \mathrm{a} \\
4,05 \mathrm{a} \\
4,30 \mathrm{a}\end{array}$ & $\begin{array}{l}6,33 \mathrm{a} \\
4,43 \mathrm{a} \\
2,35 \mathrm{a} \\
7,50 \mathrm{a} \\
8,45 \mathrm{a}\end{array}$ & $\begin{array}{r}8,49 \mathrm{a} \\
4,95 \mathrm{a} \\
10,47 \mathrm{a} \\
13,00 \mathrm{a} \\
8,44 \mathrm{a}\end{array}$ \\
\hline $\mathrm{CV}(\%)$ & $\begin{array}{r}0 \\
150 \\
300 \\
450 \\
600\end{array}$ & $\begin{array}{r}4,26 \mathrm{a} \\
6,23 \mathrm{a} \\
4,78 \mathrm{a} \\
4,78 \mathrm{a} \\
4,53 \mathrm{a} \\
36,13\end{array}$ & $\begin{array}{c}6,58 \mathrm{a} \\
7,96 \mathrm{a} \\
6,38 \mathrm{a} \\
6,78 \mathrm{a} \\
7,95 \mathrm{a} \\
58,22\end{array}$ & $\begin{array}{c}4,95 \mathrm{a} \\
4,32 \mathrm{a} \\
5,08 \mathrm{a} \\
5,03 \mathrm{a} \\
5,18 \mathrm{a} \\
31,97\end{array}$ & $\begin{array}{r}5,55 \mathrm{a} \\
4,42 \mathrm{a} \\
5,10 \mathrm{a} \\
5,55 \mathrm{a} \\
5,73 \mathrm{a} \\
18,68\end{array}$ & $\begin{array}{l}5,63 \mathrm{a} \\
4,06 \mathrm{a} \\
4,83 \mathrm{a} \\
6,23 \mathrm{a} \\
6,10 \mathrm{a} \\
22,19\end{array}$ & $\begin{array}{r}3,27 \mathrm{a} \\
1,33 \mathrm{a} \\
2,81 \mathrm{a} \\
4,37 \mathrm{a} \\
3,15 \mathrm{a} \\
16,83\end{array}$ & $\begin{array}{r}9,76 \text { a } \\
11,68 \mathrm{a} \\
11,02 \mathrm{a} \\
7,26 \mathrm{a} \\
7,36 \mathrm{a} \\
13,84\end{array}$ \\
\hline
\end{tabular}

(1) Valores médios seguidos de letras minúsculas iguais na coluna, para cada classe de tamanho de agregado e cada profundidade, não diferem estatisticamente entre si pelo teste de Tukey a $5 \%$. 
Outro fator que pode ter influenciado os resultados é o período entre a última aplicação da vinhaça e as avaliações, que foi de oito meses, o qual pode não ter sido suficiente para que ocorressem modificações significativas na estrutura do solo.

\section{CONCLUSÃO}

As doses aplicadas de vinhaça não promoveram mudanças significativas nos diâmetros médios ponderado e geométrico e no índice de estabilidade de agregados de amostras das camadas de $0-10,10-20$, 20-30 e 30-40 cm do Latossolo Vermelho distroférrico típico textura muito argilosa.

\section{LITERATURA CITADA}

AGUIAR, M.A. Incorporação de vinhaça ao solo: Efeitos sobre as características de resistência do material obtido. Campinas, Universidade Estadual de Campinas, 1992. 74p. (Tese de Mestrado)

AGUIAR, M.A.; FREIRE, W.J. \& ALBUQUERQUE, P.J.R. Caracterização física e química de dois solos tratados com vinhaça. In: CONGRESSO BRASILEIRO DE ENGENHARIA AGRÍCOLA, 21.; SIMPÕSIO DE ENGENHARIA AGRÍCOLA DO CONE SUL, 1.; Santa Maria, 1992. Anais. Santa Maria, Sociedade Brasileira de Engenharia Agrícola, 1992. p.1067-1077.

BAVER, L.D. \& GARDNER, W.H. Soil physics. 4.ed. New York, John Wiley, 1972. 498p.

BAVER, L.D.; GARDNER, W.H. \& GARDNER, W.R. Física de suelos. Barcelona, Union Tipográfica Editorial HispanoAmericana, 1973. 529p

CAMARGO, O.A.; VALADARES, J.M.A.S. \& GERALDI, R.N Características químicas e físicas de solo que recebeu vinhaça por longo tempo. Campinas, Instituto Agronômico, 1983. 30p. (Boletim Técnico, 76).

CAMPOS, B.C.; REINERT, D.J.; NICOLODI, R.; RUEDELL, J. \& PETRERE, C. Estabilidade estrutural de um Latossolo Vermelho-Escuro distrófico após sete anos de rotação de culturas e sistemas de manejo de solo. R. Bras. Ci. Solo, 19:121-126, 1995.

CARPENEDO, V. \& MIELNICZUK, J. Estado de agregação e qualidade de agregados de Latossolos Roxos, submetidos a diferentes sistemas de manejo. R. Bras. Ci. Solo, 14:99105,1990

CASTRO FILHO, C. \& LOGAN, T.J. Liming effects on the stability and erodibility of some Brazilian Oxisols. Soil Sci. Soc. Am. J., 55:1407-1413, 1991.

CASTRO FILHO, C.; MUZILli, O. \& PODANOSCH, A.L. Estabilidade dos agregados e sua relação com o teor de carbono orgânico num Latossolo Roxo distrófico, em função de sistemas de plantio, rotações de culturas e métodos de preparo das amostras. R. Bras. Ci. Solo, 22:527$538,1998$.

CINTRA, F.L.D. Caracterização do impedimento mecânico em Latossolos do Rio Grande do Sul. Porto Alegre, Universidade Federal do Rio Grande do Sul, 1980. 89p. (Tese de Mestrado)
EMPRESA BRASILEIRA DE PESQUISA AGROPECUÁRIA EMBRAPA. Centro Nacional de Pesquisa de Solos. Sistema brasileiro de classificação de solos. Brasília, Serviço de Produção de Informação, 1999. 412p.

INSTITUTO AGRONÔMICO DO PARANÁ - IAPAR. Cartas climáticas do Paraná. Disponível em: <http:// www.iapar.br>. Acesso em 16 maio 2004.

KEMPER, W.D \& CHEPIL, W.S. Size distribution of aggregates. In: BLACK, C.A., ed. Methods of soil analysis. Madison, American Society of Agronomy, 1965. p.499-510.

KEMPER, W.D. Aggregate stability. In: BLACK, C.A., ed. Methods of soil analysis. Madison, American Society of Agronomy, 1965. p.511-519.

KIEHL, E.J. Fertilizantes orgânicos. São Paulo, Agronômica Ceres, 1985. 479p.

KIEHL, E.J. Manual de edafologia: Relações solo-planta. São Paulo, Agronômica Ceres, 1979. 263p.

LONGO, R.M. Efeito da vinhaça "in natura" e biodigerida em propriedades de um solo cultivado com cana-de-açúcar. Campinas, Universidade Estadual de Campinas, 1994. 98p. (Tese de Mestrado)

LONGO, R.M.; BONI, N.R. \& ESPÍNDOLA, C.R. Efeito da vinhaça "in natura" e biodigerida em propriedades físicas de um solo cultivado com cana-de-açúcar. In: CONGRESSO BRASILEIRO DE ENGENHARIA AGRÍCOLA, 25., Bauru, 1996. Resumos. Bauru, Universidade Estadual Paulista/Sociedade Brasileira de Engenharia Agrícola, 1996. p.10.

MEDINA, C.C. \& BRINHOLI, O. Uso de resíduos agroindustriais nas produções de cana-de-açúcar, açúcar e álcool. Pesq. Agropec. Bras., 33:1821-1825, 1998.

PROGRAMA NACIONAL DE MELHORAMENTO DA CANADE-ACUÚCAR. Novas variedades RB para a região Centro Sul do Brasil. Piracicaba, 1988. p.16-20.

RESENDE, J.O. Conseqüências da aplicação de vinhaça sobre algumas propriedades físicas de um solo aluvial (estudo de um caso). Piracicaba, Escola Superior de Agricultura "Luiz de Queiroz", 1979. 112p. (Tese de Doutorado)

SANTOS, J.C.F. Comportamento de propriedades físicas e químicas de dois Latossolos roxos sob diferentes sistemas de rotação de culturas em plantio direto. Lavras, Escola Superior de Agricultura de Lavras, 1993. 101p. (Tese de Doutorado)

SILVA, I.F. Efeitos de sistemas de manejo e tempo de cultivo sobre propriedades físicas de um Latossolo. Porto Alegre, Universidade Federal do Rio Grande do Sul, 1980. 76p. (Tese de Mestrado)

SILVA, I.F. \& MIELNICZUK, J. Ação do sistema radicular de plantas na formação e estabilização de agregados do solo. R. Bras. Ci. Solo, 21:113-117, 1997.

TISDALL, J.M. \& OADES, J.M. Organic matter and waterstable aggregates in soil. J. Soil Sci., 33:141-163, 1982.

YODER, R.E. A direct method of aggregate analysis of soils and a study of the physical nature of erosion losses. J. Am. Soc. Agron., 28:337-351, 1936. 